\title{
A survey of electronic medication management technologies among nurse educators: Implications for practice
}

\author{
Eileen Creel ${ }^{* 1}$, Ann Carruth ${ }^{2}$, Staci Taylor ${ }^{1}$ \\ ${ }^{1}$ School of Nursing, Southeastern Louisisana University, United States \\ ${ }^{2}$ College of Nursing and Health Sciences, Southeastern Louisiana University, United States
}

Received: March 30, 2020

DOI: $10.5430 /$ jnep.v10n9p21

\author{
Accepted: May 20, 2020 \\ Online Published: May 24, 2020 \\ URL: https://doi.org/10.5430/jnep.v10n9p21
}

\begin{abstract}
Supervised clinical training is an essential component of nursing education programs. There has been little written about the impact of transitioning from the pre-digital healthcare environment to electronic medication management on the clinical education of nursing students. Even less is known about how nursing faculty and students incorporate technologies such a bar code medication administration into workflow to ensure correct medication administration. This article reports the results of a national descriptive cross-sectional survey of Associate and Bachelor of Science Nursing programs. Findings from this study confirm that despite the benefits of safety technologies, current practices in many clinical agencies create new challenges for nurse educators who supervise nursing students, potentially resulting in medication errors and false documentation. Nursing students in a clinical setting need to administer medications to become proficient and safe, and access to these technologies is essential. To ensure patient safety standards and legal compliance, clinical training site agencies need policies to define acceptable workflows while providing students with the practical experience needed to achieve skill competence.
\end{abstract}

Key Words: Nursing education, Technology, Bar code medication administration, Documentation

\section{INTRODUCTION}

The prevalence of medical errors was first brought to the public's attention in 1999, when the Institute of Medicine's (IOM), To Err is Human reported between 44,000 to 98,000 patients died in hospitals in the United States due to medical error, 7000 caused by medication errors. ${ }^{[1]}$ A later special report on medication safety from the IOM revealed that approximately 1.5 million preventable adverse drug events occur annually in the U. S., resulting in a total cost of $\$ 3.5$ billion. ${ }^{[2]}$ Patients also recognize safety issues with the medicationuse system. In the 2002 Commonwealth Fund Survey, 11\% of Canadian patients reported that they had been given the wrong medication at one time or another. ${ }^{[3]}$ More recently, Makary and Daniel asserted that fatal medical errors in the United States (U.S.) might be as high as a quarter million per year and could be the third leading cause of death. ${ }^{[4]}$

One-third of all medical errors which caused harm to patients in hospitals occur in the medication preparation and administration phase, which is predominantly a nursing activity. ${ }^{[5]}$ Nurses are implicated in 26\%-38\% of in-hospital medication administration errors reported. ${ }^{[6,7]}$

Nursing students are trained to practice the " 8 rights" of medication administration: right patient, right drug, right dose,

*Correspondence: Eileen Creel; Email: ecreel@ southeastern.edu; Address: School of Nursing, Southeastern Louisisana University, United States. 
right time, right route, right reason, right documentation, and right response to ensure safe medication preparation and administration. When a medication event occurs, this is often due to a breach of one of the " 8 rights". Technology has been developed to verify medications by incorporating safety technology within an electronic health record (EHR) to help prevent such errors.

Redesigning of the medication administration process, using technological advances in medication dispensing machines, Bar Coded Medication Administration (BCMA) systems, and the electronic Medication Administration Record (eMAR) have significantly reduced medication errors in clinical settings. ${ }^{[8-10]}$ Although not uniform, EHRs have become widely adopted to make patient history and care information accessible to health care providers where and when it is needed. These systems have been designed to increase quality improvement indicators consistent with the " 8 rights" of medication administration.

Implementation of BCMA presents both technical and administrative challenges. As the final delivery point for the patient, nurses are vital in ensuring the safe administration of medications. Researchers estimate that up to $25 \%$ of nurses' time is spent on activities related to medication administration. ${ }^{[11]}$ Nurse educators supervising students have the additional legal responsibility of complying with the legal authorization of pre-licensed nursing students to practice in clinical agencies. Worldwide, it is typical for nursing students to only be authorized to administer medications if they are under the supervision of an authorized person, employed in a relevant occupation. ${ }^{[12]}$

Therefore, compliance with teaching technology protocols is an essential first step in addressing on-going work processes to assure patient safety. Very little is known about behaviors of faculty and students with BCMA use. However, a review of the literature indicated that studies conducted on rates of medication errors following implementation of BCMAs did not evaluate user compliance with protocols. ${ }^{[8]}$ Introduction of technology in the clinical environment requires workflow changes. Analysis of practice processes indicated that the lack of workflow redesign and process training resulted in nurses adopting unsafe "workarounds" following BCMA implementation. ${ }^{[4,13]}$ Additionally, several studies have highlighted certain unintended consequences of its implementation, with some users either bypassing this technology or relying on it too much, thus increasing the risk of new errors. ${ }^{[15-19]}$ These are often inter-related.

Supervised clinical training is an essential component of nursing education programs providing sufficient real-world experience to assure the development of clinical competency skills. There has been little written about the impact of transitioning from pre-digital healthcare environment (a.k.a. paper-based record keeping processes and "med carts") to digital health programs or electronic medication management on the clinical education of nursing students. Even less is known about how nursing faculty and students incorporate several technologies into the workflow on units to ensure that the correct medication is administered at the correct dose at the correct time to the correct patient.

With increased utilization of technology to reduce medication errors, many schools of nursing have undertaken significant revisions to curricula and educational delivery practices to respond to the changes brought about by the increased prevalence of these technologies. Although there has been a significant change in healthcare delivery, the evolution of nurse educators in aligning and adopting technology to the clinical setting is unknown. Challenges related to electronic medication management systems include continual ongoing training for faculty and students who need to stay abreast of changing technologies in varying clinical sites.

The Quality and Safety Education for Nurses (QSEN) project identified six core competencies that schools of nursing should address in pre-licensure training to ensure that future nurses can safely perform basic practice skills. ${ }^{[20]}$ These patient care competencies include nursing students' knowledge of safety-enhancing technologies, such as medication dispensing systems, BCMA systems, and eMAR documentation. Nursing students must experience practical clinical processes in health care settings to develop the skills required for patient care and safety, including medication administration. Faculty instruction and supervision of students' use of clinical technology must conform to various training site policies to ensure the quality of patient care and safety.

Very little has been written about nurse educators' supervisory role in the retrieval of medication, BCMA use, student use of BCMA, or documentation in the EHR. Further no best practice guidelines for clinical faculty are available. A survey by researchers indicated that legal requirements surrounding nursing students' medication administration are not being met. ${ }^{[21]}$ New technology requires greater effort by clinical training site agencies to provide access to individuals from educational institutions. However, agency policies regarding the use of electronic medication administration systems by faculty and students may be inadequate, non-existent, or the clinical faculty may not be familiar with them. More information about the extent to which agencies ensure an environment for clinical faculty and students to appropriately, safely, and legally retrieve, administer, and evaluate medication administration is needed. 
A study of medication administration workflow revealed that errors are more likely to occur when there is a lapse in the critical thinking path related to the " 8 rights" of medication safety. ${ }^{[11]}$ In a dynamic clinical environment, faculty must attend to multiple direct care, teaching, and administrative functions. Little is known about the use of technology, and its impact on drug errors or near misses among nursing students. Supervision of students using technology may substantially increase time devoted to the medication administration process and increase the opportunity for interruptions and distractions during workflow.

Literature evaluating nursing students' education to assure readiness for clinical placement including preparation to function effectively with electronic medication administration systems is lacking. Krautscheid et al initiated a qualitative study in response to the number of documented medication errors and near-miss events that were not documented during senior nursing students' clinical rotation. ${ }^{[13]}$ In that study, nursing students consistently reported that theorybased training (lecture and lab), faculty demonstrations and role-modeling in the lab, as well as peer learning with practice, provided essential knowledge for clinical placement. Students also identified their unmet need to receive training in medication and documentation technologies prior to clinical placement. The study authors further reported that despite extensive laboratory practice, outdated medication administration procedures and equipment did not prepare the students to function effectively in the acute care environment. Unlike the lab, there are many distractions and interruptions in dynamic acute care settings. In addition, BCMA equipment models vary among the clinical settings that serve as nurse training sites. Even with laboratory simulation programs, students and their supervising faculty may encounter equipment with which they are unfamiliar and for which they have not received specific training.

\section{MethodS}

A cross-sectional survey design was used in this study. A survey of nursing education faculty was conducted to explore nurse educator processes and practices impacting clinical nursing students' experiences with the administration of medications using various electronic medication management system technologies such as medication dispensing, BCMA, and eMAR systems. The survey was created using the first five steps outlined by Artine et al. and consisted of demographic data and 25 questions derived from 1.) a comprehensive literature search on quality indicators of safe medication administration using technology and 2.) input from expert nurse educators. ${ }^{[22,23]}$

Demographic questions included: gender, ethnicity, level Published by Sciedu Press of education, the number of students each nurse educator supervised in a clinical setting, and the academic level of the nursing student. Clinical questions addressed: the average number of patients each student was assigned, the average number of medications each student administered per shift, the most common electronic health record vendor used, the extent of access the faculty and students had to medication administration systems, questions of process in retrieving and administering medications using technology, the expectation of medication reconciliation at end of the clinical shift, potential workaround situations using technology, and the challenges of using technology.

The survey was circulated to five nurse educators for preliminary review of content comprehensiveness and intelligibility. Based on expert feedback, modifications of the question wording and content were made. After three rounds of review, the panel of nurse educators achieved consensus on the appropriateness of questions related to student clinical experiences with medication retrieval and administration using technology in clinical settings.

Prior to conducting the study the University's Institutional Review Board approval was granted. A target population of nurse educators was recruited via randomly selected publicly identified listservs of deans and directors of associate (AD) and baccalaureate (BSN) programs accredited by Accreditation Commission for Education in Nursing or Commission on Collegiate Nursing Education. These listservs provided a sampling frame of $556 \mathrm{AD}$ programs in 46 states and 788 BSN programs in 43 states from which $20 \%$ were randomly selected. Deans and directors were asked via email invitation to forward the online survey to all clinical nurse educators. The survey invitation represented all regions of the U.S. The ability to determine the final number to whom the survey was forwarded by deans and directors was not feasible for this study design.

The survey was distributed electronically to the identified

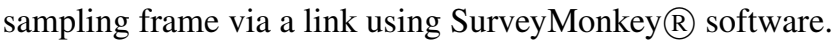
A letter of intent contained within the body of the email described the purpose of the survey and ensured anonymity of all responses. Return of the survey implied consent to participate in the study. Two-hundred thirteen nurse educators responded. Descriptive statistics were calculated using the statistical software package SPSS 23 (IBM SPSS 23.0. For MAC, SPSS Inc., Chicago, IL, USA).

\section{RESULTS}

Two-hundred thirteen $(\mathrm{N}=213)$ surveys were returned from nurse educators in 17 states representing all regions of the U.S. Responding nurse educators were equally split between 
$\mathrm{AD} /$ diploma (54\%) and BSN (46\%) programs. The majority of participants were female (92.4\%), between the ages 51-65 (52.4\%), with 6-15 years of teaching experience (39.9\%). Roughly one-third $(36.2 \%)$ of participants reported fewer than five years of teaching experience. Consistent with national patterns, survey respondents with a master's degree or higher transitioned to nursing education later in their career trajectory. Most nurse educators reported teaching six to eight nursing students (54.9\%) in mid-level curriculum placement $(42.3 \%)$ caring for one patient $(50.1 \%)$. The EHR vendors most often used by respondents were Epic (34.3\%) and Meditech (10.5\%).

The findings revealed that most clinical training site agencies do not provide nursing students with access codes needed to retrieve medication from the dispensing machine $(n=151$, $71.0 \%)$. In the majority of cases, nurse educators $(n=119)$ or staff $(n=37)$ retrieved medications, then handed them off to nursing students for administration. In those agencies where nursing students had direct access to medication dispensing machines, 33\% $(\mathrm{n}=70)$ of nurse educators indicated that nursing students had a medication error or near miss drug error using a BCMA system. Of those events, various factors were involved; in some cases, more than one factor was reported by a single respondent, thus the total percentages reported were greater than 100 . Forty-seven percent (47\%) of errors/near misses related to the nursing student's failure to use technology appropriately; $40 \%$ related to failure to comply with the "8 rights" of medication administration; $35.2 \%$ related to disruptions in the medication administration process; $22.8 \%$ related to dispensing errors in pharmacy, and $13.6 \%$ were overriding medication error alerts.

Unlike the ability to retrieve medications, slightly more than half of the nurse educators reported that nursing students (60\%) were assigned an EHR code allowing them to chart medication administration in the patient's eMAR. However, additional questions revealed that even when nursing students had EHR access codes, they frequently did not document medication administration. Nurse educators reported that fewer than half (47.4\%) of nursing students documented the administration of medication in the eMAR either independently, with a faculty member, or with staff. Co-signing the eMAR documentation of a student who administered medication by the faculty was required less than $50 \%$ of the time ( $\mathrm{n}=96$ ). Most concerning, $40.8 \%$ of nurse educators used their personal access code/badge to document medication administration that was in fact given by nursing students. A limited number of students independently scanned and documented administration of medications (8.5\%). Six and a half percent $(6.5 \%)$ of staff nurses used their access code to document medication administered by the nursing students.
The majority of respondents (74\%) believed technology has improved patient safety. However, $26 \%$ indicated the availability of technology (e.g., hand-held scanners) was often inadequate to meet student needs on the units.

Technological issues arose during supervision of medication administration; $50 \%$ of nurse educators reported a need to request over-rides because medication labels were not readable and $10 \%$ indicated they needed to perform over-rides because patients' armbands were not scannable. Sixteen percent $(16 \%)$ of nurse educators indicated they felt that technology "workarounds" were inevitable because of time constraints.

\section{Discussion}

While there have been nursing education studies focusing on medication administration and reasons for errors, very few have focused on the nurse educators' perceptions of practices and processes. A systematic review by Gaard and Orbaek focused on staff, not nurse educators, supervising students with technology. ${ }^{[24]}$ This is the first such study describing the experiences of nurse educators with the complex technologydriven health systems requiring supervision of students with dispensing, administration, and documentation. The foundation of nursing documentation is "if it is not documented, it is not done". Documentation is an essential professional and legal requirement of nursing practice and is consistently cited in the worldwide nursing codes of ethics or codes of conduct. Therefore, the findings of this study note several practice and safety concerns for nurse educators.

In their development of a BCMA evaluation process Kelly et al. stated that workarounds are most commonly developed as solutions to barriers in patient care delivery, to account for a technology shortcoming, or in response to time constraints. ${ }^{[22]}$ Koppel et al. identified and categorized the types and frequency of nursing workarounds following BCMA implementation. ${ }^{[17]}$ They identified 31 discrete situations that potentially cause nurses to deviate from BCMA protocol steps and observed 15 workarounds often used by nurses. Analysis of actual work flow processes categorize to group potential workaround situations into three broad categories: omission of process steps, steps performed out of sequence, and unauthorized process steps. ${ }^{[17]}$ A survey of acute care nurses was conducted by Rack et al. to ascertain their perceptions of how often they use workarounds. ${ }^{[14]}$ The study found that the majority of nurses surveyed engaged in a workaround during their last shift worked. Sixty-three percent administered a medication without scanning the armband, $72 \%$ scanned the medication after it was administered, and 23\% put a patient armband on another object to scan. Faculty and students are expected to follow policies related to BCMA 
and EHR. Yet, when the environment lacks sufficient support for educators it is possible patient safety is jeopardized. No research to date has described various indicators of the "8 rights" as it relates to the technology acquisition, uses, or potential system overrides related to system failures among nursing faculty and students in clinical settings.

In this study, over $55 \%$ of the nurse educators reported retrieving medications from dispensing medication machines for nursing students because the students did not have their own access code. When faculty retrieve medications and hand off to the nursing students to administer, this results in an out of sequence workaround. Future research needs to explore the extent to which nurse educators and staff engage in omissions of process (incorrect documentation) and the potential impact on patient safety when handing medications off to another person without verification. While unimaginable for some faculty, for some state boards of nursing this may violate the nurse practice act.

Additionally, nurse educators reported varying levels of proficiency in accessing and documenting medication administration and patient care. Forty percent (40\%) of nurse educators routinely scanned the medication in BCMA/eMAR, therefore, recording the faculty name as having administered the medication, when in reality it was the nursing student who administered. Future research should explore the extent to which EHRs allow alternate recording of the nursing student's name (e.g., in a comment box or as a co-administrator). A comment box would allow for accurate documentation that the medication was administered in fact by the student, not the person with the access code or badge (e.g., nurse faculty). A record that reflects a nurse faculty's access code despite the fact that they did not administer the medication would result in inaccurate and false documentation. In the event of variance, or adverse reaction, the nurse educator is at legal risk of not only falsely documenting in the record, he/she is also inadequately supervising students and may be subject to disciplinary action based on state practice act governing nursing education and supervision.

Contributing factors to medication errors and near misses included failure to use technology appropriately and overriding medication error alerts. Overrides may occur with situations where scanning interrupts successful workflow. Instead of following the scanning policy, nurses resort to manually entering medication administration information bypassing the technological barrier. When faculty override BCMA safety alerts to meet patient care or student training needs, an unauthorized process step occurred. Studies have identified technology as a concern for nursing students training in simulated experiences. ${ }^{[25]}$ Additionally, workarounds

Published by Sciedu Press could result in an omitted medication or medication being documented as administered at the wrong time. ${ }^{[17]}$

Findings from this study confirm that despite the benefits of safety technologies, current practices in many clinical agencies create new challenges for nurse educators who supervise nursing students, potentially resulting in medication errors and false documentation. It stands to reason if agencies want a work-ready workforce, senior nursing students (those in their last year or last semester of clinical practice) may be more likely than lower level students to be issued an individual bar code access badge or some other unique access code. Regardless of individual student access to BCMA or eMAR, most nursing students in a clinical setting need to administer medications to become proficient and safe.

This study has several limitations. The results using survey data rely on self-reported experiences, which have an inherent error in memory and potential for bias. While survey returned represent all regions of the U.S., representation of nurse educators is unknown. The survey failed to ask for input from faculty related to their expertise in the use of or experience with electronic medication administration systems. This study focused on nurse educators supervising nursing students in acute clinical settings only, and therefore, may not resonate with educators in long-term care or community clinical settings. Many schools of nursing use employed nurse to preceptor for training of nursing students in their final semesters. Nurse preceptors were not surveyed in this study. Future research should compare technical "saviness" and institutional familiarity with likelihood to engage in unsafe work arounds. Additionally, participant perceptions are based on diverse real-world experiences and a variety of different curricula throughout the nation. Therefore, while processes of safe medication administration education is expected across all nursing programs, the findings might be limited due to experience variations and the length of time and adequacy of training on use of technology in each facility.

\section{Conclusions}

With the increasing use of electronic health technology in clinical practice, it is essential that nurse educators develop deeper understandings of how technologies like BCMA and eMAR can be integrated meaningfully into nursing education. Based on the results of this national survey, it is clear that nurse educators who supervise nursing students' medication administration need to know and consistently model competence with clinical technology protocols. Nurse educators, directly supervising nursing students, must competently and accurately incorporate the patient safety considerations of the "8 rights" while using technology to dispense, ad- 
minister, and document patient care. For example, nursing faculty must additionally ensure training for student nurses includes concepts, such as the "rights" associated with the right reason for administration of the medication and the "right" response. [26]

Health care systems must partner with nurse educators to ensure competence in the use of all medication dispensing, BCMA, eMAR, and EHR systems for which they are supervising nursing students. Partnering should include hands-on experience during simulation as well as real-world clinical experiences. Kelly et al. indicated that to create a culture of safety pro-actively requires ongoing evaluation and adjustments of EHR systems with input from front line users. ${ }^{[22]}$ For agencies serving as clinical training sites, frontline users of EHR systems include nurse educators and the nursing students they supervise. Thus, they should be included in providing input to prevent the need for overrides and workarounds with aquipment.

\section{CONFLICTS OF INTEREST Disclosure}

The authors declare that there is no conflict of interest.

\section{REFERENCES}

[1] Institute of Medicine. To err is human: Building a safer health system. Washington, DC: National Academy Press; 2000.

[2] Institute of Medicine. Preventing medication errors. Washington, DC: The National Academies Press. 2007.

[3] Schoen C, DesRoches C, Downey D. The Canadian health care system: Views and experiences of adults with health problems. New York, NY: The Commonwealth Fund. 2003.

[4] Makary MA, Daniel M. Medical error-the third leading cause of death in the U.S. British Medical Journal. 2016; 353: i2139. PMid:27143499 https ://doi.org/10.1136/bmj .i2139

[5] Barker KN, Flynn EA, Pepper GA, et al. Medication errors observed in 36 health care facilities. Achieves of Internal Medicine. 2002; 162: 1897-1903. PMid:12196090 https://doi.org/10.1001/ar chinte.162.16.1897

[6] Bates DW. Preventing medication errors: a summary. American Journal of Health-System Pharmacy. 2007; 64(14 Suppl 9): S3-S9. PMid:17617512 https : //doi.org/10.2146/ajhp070190

[7] Weeks KW, Sabin M, Pontin D, et al. Safety in numbers: an introduction to the nurse education in practice series. Nurse Education Practice. 2013; 13: e4-e10. PMid:22795760 https ://doi .org/10 $.1016 / j . n e p r .2012 .06 .006$

[8] Hassink J, Jansen M, Helmons P. Effects of bar code-assisted medication administration (BCMA) on frequency, type and severity of medication administration errors: A review of the literature. European Journal of Hospital Pharmacy. 2012; 19: 489-494. https: //doi.org/10.1136/ejhpharm-2012-000058

[9] Keohane CA, Bane AD, Featherstone E, et al. Quantifying nursing workflow in mediation administration. Journal of Nursing Administration. 2008; 38: 19-26. PMid:18157001 https://doi.org/10.1 097/01. NNA.0000295628.87968.bc

[10] Poon E, Keohane C, Yoon C, et al. Effect of bar-code technology on the safety of medication administration. New England Journal of Medicine [Internet]. 2010; 362: 1698-707. PMid:20445181 https://doi.org/10.1056/NEJMsa0907115

[11] Keers R, Williams S, Cooke J, et al. Causes of medication administration errors in hospitals: A systematic review of quantitative and qualitative evidence. Drug Safety. 2013; 36: 1045-1067. PMid:23975331 https://doi.org/10.1007/s40264-013-0090-2

[12] Reid-Searl K, Happell B, Burke K, et al. Nursing students and the supervision of medication administration. Collegian. 2013; 20 : 109. PMid:23898599 https://doi.org/10.1016/j.colegn.2 012.04 .003
[13] Krautscheid LC, Orton VJ, Chorpenning L, et al. Student nurse perceptions of effective medication administration education. International Journal of Nursing Education Scholarship. 2011; 8: 1-15. https://doi.org/10.2202/1548-923X. 2178

[14] Rack LL, Dudjak LA, Wolf GA. Study of nurse workarounds in hospital using bar code medication administration system. Journal of Nursing Care Quality. 2012; 27: 232-239. PMid:22202186 https ://doi.org/10.1097/NCQ.0b013e318240a854

[15] Cochran GL, Jone KJ, Brockman J, et al. Errors prevented by and associated with bar-code medication administration systems. Joint Commission Journal of Quality Patient Safety. 2007; 33: 293-301. https://doi .org/10.1016/S1553-7250(07) 33034-1

[16] DiConsiglio J. Creative 'work-arounds' defeat bar-coding safeguard for meds: study finds technology often doesn't meet the needs of nurses. Materials Management in Health Care. 2008; 17: 26-9.

[17] Koppel R, Wetterneck T, Telles JL, et al. Workarounds to barcode medication administration systems: their occurrences, causes, and threats to patient safety. Journal of American Medical Informatics Association. 2008; 15: 408-23. PMid:18436903 https: //doi.org/10.1197/jamia.M2616

[18] Rivish VO, Modeda MD. Medication administration pre and post BCMA at the VA Medical Center. Online Journal of Nursing Informatics. 2010; 14: 1-21.

[19] Seibert HH, Maddox RR, Flynn EA, et al. Effect of barcode technology with electronic medication administration record on medication accuracy rates. American Journal of Health-System Pharmacy. 2014; 71: 209-218. PMid:24429014 https://doi.org/10.2146/ajhp 130332

[20] The QSEN Institute. QSEN competencies. [Internet]. 2019. Available from: http://qsen.org/competencies/pre-licensure-ksa s/\#safety

[21] Reid-Searl K, Moxham L, Walker S, et al. Supervising medication administration by undergraduate nursing students: Influencing factors. Journal of Clinical Nursing. 2010; 19: 775-784. PMid:20500321 https://doi.org/10.1111/j.1365-2702.2009.03074.x

[22] Kelly K, Harrington L, Matos P, et al. Creating a culture of safety around bar-code medication administration: an evidence-based evaluation framework. Journal of Nursing Administration. 2016; 46: 30-7. PMid:26641468 https://doi.org/10.1097/NNA. 0000000000 000290

[23] Artino AR, La Rochelle JS, Dezee KJ, et al. Developing questionnaires for educational research: AMEE Guide No. 87. Medical Teacher. 2014; 36(4): 63-474. PMid:24661014 https : //doi .org/ 10.3109/0142159X. 2014.889814 
[24] Gaard M, Orbaek J. Supervising nursing students in a technologydriven medication administration process in a hospital setting: a systematic review protocol. Joanna Briggs Institute. 2016; 14: 52-57. PMid:27635745 https://doi.org/10.11124/JBISRIR $-2016-003051$

[25] Booth R, Sinclair B, Strudwick G, et al. Identifying error types made by nursing students using eMAR technology. Clinical Simulation in
Nursing. 2017; 13: 492-500. https://doi.org/10.1016/j.ec ns.2017.05.016

[26] Huges RG. Medication administration safety in: Huges, R.G (ED.). Patient safety and quality: an evidence-based handbook for nurses. 2008. Rockville, MD: Agency for Healthcare and Research Quality; 2008. 397-457 pp. 